\title{
Correlation of the Neutrophil Lymphocyte Ratio with Malignancy Status in Thyroid Nodule
}

\author{
Agus Maulana $^{1}$, Dwi Hari Susilo ${ }^{2}$, Marjono Dwi Wibowo ${ }^{2}$ \\ ${ }^{1}$ Resident of General Surgery, Department of Surgery Dr. Soetomo Hospital, Faculty of Medicine, Airlangga \\ University Surabaya, East Java 60285, Indonesia \\ ${ }^{2}$ Senior Staff, Head and Neck Division, Department of Surgery Dr. Soetomo Hospital, Faculty of Medicine, \\ Airlangga University Surabaya, East Java 60285, Indonesia
}

Corresponding Author: Agus Maulana

\begin{abstract}
Background: Thyroid nodules are very common, found in more than $50 \%$ of patients when using ultrasonography. While the prevalence of thyroid nodules in the population is increasing, only $5 \%$ to $10 \%$ harbor malignant disease. Still, there is a pressing need to correctly identify the nature of thyroid nodules, because thyroid cancer is the most common endocrine malignancy and often presents in thyroid nodules. Inflammation plays a critical role in many aspects of cancer, including tumor development, progression, clinical presentations, and prognosis. The neutrophil/ lymphocyte ratio (NLR) is a simple index of systemic inflammatory response ${ }^{1,2}$. The aim of this study is to determine whether there is a relationship between the neutrophil/lymphocyte ratio with malignancy status of thyroid nodules.

Methods: One hundred two cases of benign and malignant thyroid nodule were analyzed retrospectively. We evaluated neutrophil lymphocyte ratio with result post-operative pathological anatomy. Complete blood counts with differential counts were taken before operation. NLR was calculated by dividing preoperative neutrophil count with lymphocyte count. Sample were categorized into low grade (NLR < 1,91) and high grade (NLR $\geq 1,92$ ).
\end{abstract}

Results: There was statistically significant difference of neutrophil-lymphocyte ratio between benign thyroid nodule and malignant thyroid nodule ( $\mathrm{p}=0,001)$.

Conclusion: NLR is easily accessible tests that can be automatically calculated from complete blood count without additional cost in differentiation of malignant thyroid nodule from benign nodule in the preoperative period.

Keywords: Thyroid nodule; neutrophil lymphocyte ratio; malignancy.

\section{BACKGROUND}

Thyroid nodule is a discrete lesion in the thyroid gland that is radiologically distinct from the surrounding thyroid parenchyma. Thyroid nodulescan be caused by many disorders: Benign (colloid nodule, Hashimoto's thyroiditis, simple or hemorrhagic cyst, follicular adenoma and subacute thyroiditis) and malignant (papillary cancer follicular cancer, Hurthle cell (oncocytic) cancer, anaplastic cancer, medullary cancer, thyroid lymphoma and metastases) ${ }^{1}$. About 230,000 new cases of thyroid cancer were estimated in 2012 among women and 70,000 among men, with an age standardized (world population) rate of $6,1 / 100,000$ women and 1,9/100,000 $\mathrm{men}^{2}$. Fine needle aspiration (FNA) cytology is generally considered the first line diagnostic test to screen for thyroid cancer. The reported sensitivity of FNA for thyroid cancer is between $84 \%$ and $93 \%$ and specificity between $75 \%$ and $99 \%^{3}$.

The mediators and cellular effectors of inflammation are important constituents of the local environment of tumours ${ }^{4}$. Tumours have several chemotaxis mechanisms in regulating neutrophil recruitment through chemokines. Once neutrophils arrive at tumour sites, they 
can be instructed by tumour- derived factors to "tune up" their supporting function.

The role of protomours neutrophils is by secreting Reactive Oxygen Species (ROS) which is known to cause DeoxyriboNucleic Acid (DNA) damage inpremalignant cell and drives oncogenic transformation, secreting proangiogenic factor Vascular Endothelial Growth Factor (VEGF) and Bv8. Neutrophils also secrete the matrix metalloproteinase and serine protease that can damage the extracellular matrix and release bioactive growth factors and proangiogenic molecules and may stimulate local immunosuppression by disrupting $\mathrm{T}$ cell responses including cell death and $\mathrm{T}$ cell recruitment, allowing growth, development and progression tumours 5 .

Neutrophil-lymphocyte ratio (NLR) of thyroid nodules is expected to assist in predicting the risk of malignancy in thyroid nodules, particularly in the results of FNA, which may help the selection of ases for surgery orconservative. In addition, NLR may also confirm benign FNA results in the thyroid, considering there are false negative values of $2 \%$ to $10 \%$. This study aims to to determine whether there is a relationship between the neutrophil/lymphocyte ratio with malignancy status of thyroid nodules.

\section{METHODS}

This study was an retrospective cross sectional study to to determine whether there is a relationship between the neutrophil/lymphocyte ratio with malignancy status of thyroid nodules conducted at Dr. Soetomo Hospital Surabaya fromJanuary, 2018 to December, 2020.

Sample in this research is all cases of thyroid nodule in Dr. Soetomo Hospital
Surabaya fromJanuary, 2018 to December, 2020 that meets the inclusion and exclusion criteria. Inclusion criteria in this research are: (1) All cases of thyroid nodules who underwent thyroid surgery at Dr. Soetomo hospital; Exclusion criteria in this research are: (1) All cases of thyroid nodules who had undergone surgery to remove the thyroid gland or had a tumor other than the thyroid. (2) All cases of thyroid nodules who have an autoimmune disease, hematological disorders, concomitant infectious diseases both acute or chronic, and undergoing steroid treatment, chemotherapy, or radiotherapy.

Dependent variable in this study is anatomic pathology on thyroid nodules based on anatomical pathology results after surgery and dependent variable is ratio neutrophil/ lymphocyte from laboratory examination which could be on examination of peripheral blood of patients undergoing surgery. The laboratory values are presented in categorical data with a cut of point value off 1,91 . NLR $<1.91$ is considered low and NLR $\geq$ 1.92 is considered high $^{6}$.

The study sample was chosen consecutively (consecutive sampling) i.e. all subjects meeting the inclusion criteria and willing to participate in the study will be included without randomization. Univariate analysis is used to describe each variable with frequency distribution table and to know the difference between variable by using chi-square test.

\section{RESULTS}

In this study, there were 112 cases which met the inclusion and exclusion criteria, according to the calculation of the sample size, the characteristics data of the research subjects can be seen in Table 1 .

Tabel 1. The characteristics data

\begin{tabular}{|c|c|c|c|}
\hline Variable & Benign thyroid nodule & Malignt thyroid nodule & p-value \\
\hline Age (years) & $48,1 \pm 11,29$ & $52,9 \pm 15,65$ & 0,014 \\
\hline Gender: Male / Female & $14(25 \%) / 42(75 \%)$ & $12(21,4 \%) / 44(78,6 \%)$ & 0,412 \\
\hline Neutrophil $\left(10^{3} \mathrm{sel} / \mu \mathrm{l}\right)$ & $4,6 \pm 1,29$ & $5,6 \pm 2,71$ & $<0,001$ \\
\hline Lymphocyte $\left(10^{3} \mathrm{sel} / \mu \mathrm{l}\right)$ & $2,4 \pm 0,52$ & $1,9 \pm 0,61$ & 0,082 \\
\hline \multirow[t]{2}{*}{ NLR } & $1,8 \pm 0,49$ & $3,2 \pm 2,19$ & 0,001 \\
\hline & & & Count(n\%) \\
\hline \multirow[t]{5}{*}{ Anatomical Pathology } & \multirow[t]{2}{*}{ Benign thyroid nodule } & Adenomatous goiter & $51(45 \%)$ \\
\hline & & Follicular adenoma & $5(4,5 \%)$ \\
\hline & \multirow[t]{3}{*}{ Malignant thyroid nodule } & Papillary thyroid carcinoma & $47(42 \%)$ \\
\hline & & Follicular thyroid carcinoma & $7(6,3 \%)$ \\
\hline & & Anaplastic thyroid carcinoma & $2(1,8 \%)$ \\
\hline
\end{tabular}


This study were found 86 thyroid nodules in women $(76,8 \%)$, with 44 sample $(39,3 \%)$ of them are malignant, and 26 thyroid nodules in men $(23,2 \%)$, with 12 sample $(10,7 \%)$ of them are malignant. In these study, benign thyroid tumor were found in 18 years old subject as the youngest while 70 years old as the oldest, and for malignant thyroid nodule, the youngest subject is 22 years old and the oldest is 81 years old.

Most primary thyroid cancers are epithelial tumors that originate from thyroid follicular cells. These cancers develop three main pathological types of carcinomas: papillary thyroid carcinoma (PTC), follicular thyroid carcinoma (FTC) and anaplastic thyroid carcinoma (ATC). Papillary thyroid carcinoma consists of 85$90 \%$ of all thyroid cancer cases growth, followed by FTC $(5-10 \%)^{7,8}$. Based on the results of postoperative anatomical pathology, collected 112 sample where most of the results of anatomic pathology are adenomatous goiter with $51(45 \%)$ sample, followed by papillary thyroid carcinoma with 47 (42\%) sample, follicular thyroid carcinoma with $7(6,3 \%)$ sample, follicular adenoma with $5(4,5 \%)$ sample, and anaplastic thyroid carcinoma with $2(1,8 \%)$ sample.

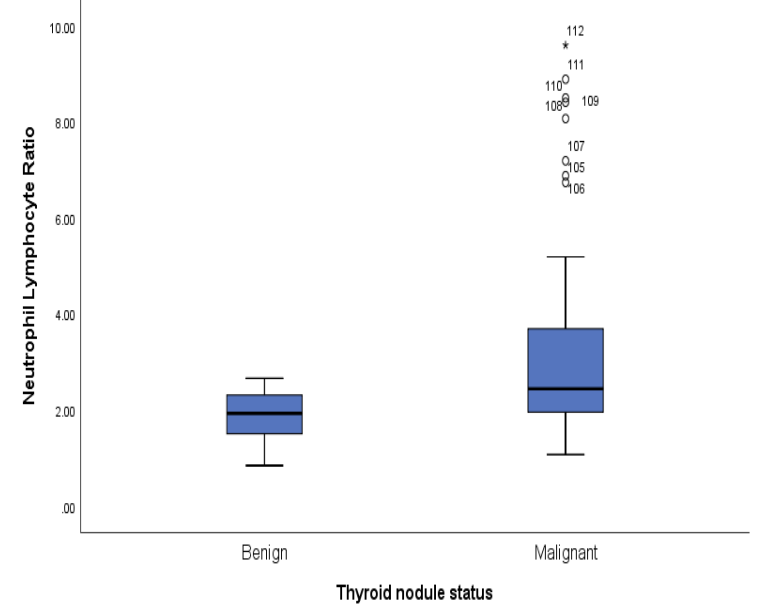

Figure 1. Mean NLR Levels. Statistical significance was obtained when comparing group Benign and Malignant thyroid nodule $(p=0.001)$

In this study, value of the high NLR $(\geq 1,92)$ were $73(65,2 \%)$ sample consisting of $28(25 \%)$ sample with benign thyroid nodule and $45(40,18 \%)$ sample with malignant thyroid nodule, while the low NLR $(<1,91)$ were $39 \quad(34,8 \%)$ sample consisting of $28(25 \%)$ sample with benign thyroid nodule and $11(9,82 \%)$ sample with malignant thyroid nodule. Sample with high NLR $(\geq 1,92)$ and malignant nodules were 45 (40,18\%) from 73 sample. The low NLR $(<1,91)$ and benign nodules were $28(25 \%)$ from 39 sample, which was statistically significant difference $(\mathrm{p}=0,001)$ figure 1 .

\section{DISCUSSION}

Thyroid nodules are one of the clinically and radiologically common pathologies. The most common type among the malignant nodules is differentiated thyroid cancer, and its incidence has been increasing throughout the world. It is important to differentiate benign and malignant thyroid nodules in $7-15 \%$ of thyroid nodules ${ }^{9}$.

Thyroid nodules and malignant thyroid nodules are more common in women. Estrogens are thought to be associated with increased incidence of thyroid nodules and thyroid cancer. The thyroid tissue express estrogen receptor, estrogen receptor $\alpha$ and $\beta$, where there is a significant increase of expression level in response to 17 $\beta$-estradiol. Estrogen receptors are intracellular receptors that serve as transcription factors. Estrogens promote the growth of benign and malignant thyroid nodules by binding to these estrogen receptor nuclei and by activating the mitogen activated protein (MAP) kinase pathway thus mediating the occurrence of mitogenesis ${ }^{10}$.

Malignancy increasing with age, most of the tumours occurs in third to sixth decade of life. This may be due to changes in normal thyroid tissue and decreased immune systems occur in line with age. Fine needle aspiration is the preferred method with good sensitivity and cost effectiveness in evaluating thyroid nodules ${ }^{12}$.

The two most frequently used markers of active inflammatory status in 
patients with cancer are C-reactive protein (CRP) and NLR, because they are reliable and widely available in daily clinical practice and their sensitivity in predicting survival rates has been supported by a large number of studies in various types of cancer $^{13}$.

Neutrophil to lymphocyte ratio has emerged as a simple and valid composite marker of systemic inflammatory response. Compared with serum CRP, which is not routinely measured as part of the cancer work up, NLR is inexpensive, easily calculated and universally available ${ }^{1}$.

The NLR is an indicator of both inflammatory burden (neutrophil count) and regulatory mechanisms (lymphocyte count) in inflammatory diseases. It is a quantitative expression of the systemic inflammatory response due to malignant tumors and reflects the relationship between tumorogenesis and chronic inflammation. High NLR may result from neutrophilia and/or lymphopenia. Neutrophils play an important role in the process of malignant transformation and metastasis. The role of neutrophils here is to support tumor growth and metastasis, due to the ability of neutrophils to produce and release growth factors such as vascular endothelial growth factor (VEGF) which together with growth factors other trigger angiogenesis and vascularisation resulting in an increase in tumor growth rate. Neutrophils also stabilize the attachment of tumor cells to the endothelium and assist the migration of tumor cells out of the vasculature. In this case, neutrophils act as pro- tumors. While the importance of lymphocytes is emphasized to tumors with high density of lymphocyte infiltration tend to have a better prognosis. Cytotoxic CD8+ T cells (CTL), natural killer (NK), and T NK cells (NKT cells) have an important role in preventing tumor growth because of their cytotoxic function and ability to induce apoptosis to destroy cancer cells. In this case, lymphocytes act as anti-tumor.

Many cancer survival studies have suggested that NLR is a significant predictor of overall. Patients with elevated NLR have a relative lymphocytopenia and neutrophil leukocytosis in favor of protumour inflammatory response, in different types of cancer. Seretis et al. compared the NLR between those with an incidental papillary microcarcinoma and those with a benign goiter only and found that the former group had a significantly higher mean NLR than the latter group $(3,0$ vs. $1,9 \mathrm{p}<0.001)$. The authors concluded that NLR could potentially be used as a biomarker for detecting incidental small PTC in an apparently benign goiter ${ }^{2}$.

In the study of Ceilan et al. concluded that high NLR correlated with tumor size and extrathyroidal extension. NLR can be used as a marker to confirm patients with papillary thyroid carcinoma (PTC). Ceilan et al. collected 201 samples of papillary thyroid carcinoma patients with a mean NLR value of 2,11 $\pm 0,94$ which were then divided into two groups using an NLR cut off value of 1,92 . Patients with NLR $<1,91$ totaled 100 samples and NLR>1,92 totaled 101 samples. There was a statistically significant difference between the two groups with tumor size $(p=0,002)$ and extra thyroid extension $(\mathrm{p}=0,028)^{13}$. Seretis et al. found high NLR values $(>2,5)$ in papillary thyroid microcarcinomas (PTMC). Seretis et al. In his study, 26 patients used benign goiter samples, and papillary thyroid microcarcinomas 31 patients with (PTMC). The NLR values in benign goiter and PTMC had a statistically significant difference $(\mathrm{p}=0.001)^{14}$. Ari et al. in research results concludes that NLR and PLR are strong parameters in both thyroiditis and papillary thyroid cancer, but NLR is superior in diagnosing thyroiditis ${ }^{15}$.

Kocer et al. demonstrate that NLR is higher in group lymphocytic thyroiditis with papillary thyroid carcinoma and group papillary thyroid carcinoma than multinodular goiter and lymphocytic thyroiditis $(\mathrm{p}<0,05)$, it may be used for be aware of presence of malignity. This study suggested that use of cut-off value of 1,91 for NLR would be optimum for clinical use 
to identify patients with PTC as sensitivity of $89 \%$ and specificity of $54,5 \%$ can be achieved. At this cut-off point negative predictive value was $92 \%$. In this study, mean NLR was found in benign thyroid nodule $39(34,8 \%)$ sample and $73(65,2 \%)$ sample in malignant thyroid nodule, which statistically significant difference $(\mathrm{p}=0,001)$. Further research is required in determining the sensitivity and specificity values. Nevertheless the use of NLR should not be considered a decisive factor but it only provides an option in strengthening the diagnosis, since histopathology is a gold standard in diagnosis.

\section{CONCLUSION}

There was statistically significant difference of neutrophil/lymphocyte ratio between benign thyroid nodule and malignant thyroid nodule.

\section{Declaration}

\section{Consent for Publication}

All participants give their consent to participate in this study and that the information will be published in this journal.

\section{Availability of Data and Material}

The datasets used and/or analysed during the current study are available from the corresponding author on reasonable request.

\section{Abbreviations:}

NLR: Neutrophil/Lymphocyte Ratio; FNA: Fine Needle Aspiration; ROS: Reactive Oxygen Species; DNA: Deoxyribo Nucleic Acid; VEGF: Vascular Endothelial Growth Factor; NK: natural killer, PTC: Papillary Thyroid Carcinoma; FTC: Follicular Thyroid Carcinoma; ATC: Anaplastic Thyroid Carcinoma; MAP: Mitogen Activated Protein; ATA: American Thyroid Association; CRP: C-Reactive Protein

\section{Competing Interest}

The authors declare that they have no competing interests.

\section{Funding}

All funding from this study come from authors its self.

\section{Author's Contributions}

The authors are agree and responsible for the content and writing of the paper. All authors have contributed significantly, read and approved the final manuscript. The authors are agree and responsible for the content and writing of the paper. All authors have contributed significantly, read and approved the final manuscript.

\section{ACKNOWLEDGMENTS}

We are gratefully indebted to our study participants in Dr. Soetomo General Hospital. We also express our gratitude to Department of Surgery Faculty of Medicine Community Universitas Airlangga and to the Health Research Ethics Committee that gave permission, license and approved this research.

\section{Ethical Approval:}

Ethics Committee Approval

Health Research Ethics Committee (HREC)

Dr. Soetomo Hospital

Ref: 0226/KEPK/VII/2021

\section{REFERENCES}

1. Liu C.L. et al. 2013. Blood neutrophil-tolymphocyte ratio correlates with tumor size in patients with differentiated thyroid cancer. Journal of surgical oncology 107 , 493-497, doi: 10.1002/jso.23270.

2. Haugen B.R. et al. 2015. Management Guidelines for Adult Patients with Tiroid Nodules and Differentiated Tiroid Cancer The American Tiroid Association (ATA) Guidelines Task Force on Tiroid Nodules and Differentiated Tiroid Cancer. American Tiroid Association;26(1);1-133

3. Tamhane S, Gharib H. 2016. Thyroid nodule update on diagnosis and management. Clin Diabetes Endocrinol; 2:17.

4. Brophy C. et al. 2015. Impact of microcalcifications on risk of malignancy in thyroid nodules with indeterminate or 
benign cytology. Otolaryngol Head Neck Surg. 2015;154:46-51.

5. Liang W, Ferrara N. 2016. The complex role of neutrophils in tumor angiogenesis and metastasis. Cancer Immunol Res. 2016;4:83-91.

6. Kocer D. et al. 2015. May the neutrophil/lymphocyte ratio be a predictor in the differentiation of different tiroid disorders? Asian Pac J Cancer Prev; 16(9):3875-9.

doi:

10.7314/apjcp.2015.16.9.3875. PMID: 25987053.

7. Katoh H. et al. 2015. Classification and general consideration of thyroid cancer. Ann Clin Pathol. 3:1045.

8. Gharib H. et al. 2010. American association of clinical endocrinologists, associazione medici endocrinologi and European thyroid association medical guidelines for clinical practice for the diagnosis and management of thyroid nodules. Endocr Pract. 1:1-43.

9. Lang B. et al. 2014. Does Preoperative neutrophil lymphocyte ratio predict risk of recurrence and occult central nodal metastasis in papillary thyroid carcinoma. World J Surg. 38:2605-12.

10. Manole D. et al. 2001. Estrogen promotes growth of human thyroid tumor cells by different molecular mechanisms. J Clin Endocrinol Metab. 86:1072-77.
11. Haymart M.R. 2009. Understanding the relationship between age and thyroid cancer. Oncologist.14:216-21.

12. Cooper D.S. et al. 2009. Revised American thyroid association management guidelines for patients with thyroid nodules and differentiated thyroid cancer. Thyroid. 19:1167-14.

13. Ceylan Y. et al. 2019. The Correlation of Clinicopathological Findings and Neutrophil-to-Lymphocyte and Platelet-toLymphocyte Ratios in Papillary Thyroid Carcinoma. Mol Imaging Radionucl Ther. Mar 19;28(1)

14. Seretis C. et al. 2013. The significance of neutrophil/lymphocyte ratio as a possible marker of underlying papillary microcarcinomas in thyroidal goiters : A pilot study. Am J Surg. 205:691-96.

15. Ari A, Gunver F. 2019. Comparison of neutrophil-lymphocyte ratio and plateletlymphocyte ratio in patients with thyroiditis and papillary tumors. J Int Med Res; 47(5):2077-83.

How to cite this article: Maulana A, Susilo DH, Wibowo MD. Correlation of the neutrophil lymphocyte ratio with malignancy status in thyroid nodule. International Journal of Research and Review. 2021; 8(9): 23-28. DOI: https://doi.org/10.52403/ijrr.20210905 